\title{
Effects of intra- and extracellular factors on anti-aging klotho gene expression
}

\author{
K. Turan ${ }^{1}$ and P. Ata ${ }^{2}$ \\ ${ }^{1}$ Department of Basic Pharmaceutical Sciences, Faculty of Pharmacy, \\ Marmara University, Haydarpasa, Uskudar, Istanbul, Turkey \\ ${ }^{2}$ Department of Molecular Genetics, \\ Haydarpasa Numune Research and Training Hospital, Uskudar, \\ Istanbul, Turkey \\ Corresponding author: K. Turan \\ E-mail: kadirturan@marmara.edu.tr
}

Genet. Mol. Res. 10 (3): 2009-2023 (2011)

Received January 3, 2011

Accepted July 26, 2011

Published September 9, 2011

DOI http://dx.doi.org/10.4238/vol10-3gmr1261

\begin{abstract}
Inactivation of the klotho gene in mice causes serious systemic disorders, resembling human aging. However, at the molecular level, its action mechanisms are not well understood. The stimulatory or inhibitory effects of cis- and trans-regulatory factors on the klotho gene expression are also still unclear. We studied the effects of intra- and extracellular factors on human klotho gene expression. For this purpose, pHKP-Luc and pHKP-GFP reporter vectors were constructed with the 2.1-kbp upstream region of human klotho, covering its promoter region, using luciferase and GFP genes as the reporter. A series of vectors that have deletions in the upstream region of the klotho gene were constructed to assay cisacting factors. Deletion of some parts of the klotho gene upstream region significantly affected reporter gene expression in HEK293 cells. p16 and p53 proteins inhibited reporter luciferase expression under the control of human klotho promoter in a dose-dependent manner. Calcium and phosphate ions stimulated klotho expression.
\end{abstract}


p21, PTH, IGF-1, and angiotensin-II had no significant effect on klotho expression in HEK293 cells.

Key words: klotho gene; Anti-aging; Apoptosis; PTH; IGF-1; Angiotensin-II

\section{INTRODUCTION}

The klotho gene was originally identified as a gene that, when insertionally inactivated in a mouse strain, causes a syndrome resembling human aging, including osteoporosis, skin atrophy, pulmonary emphysema, atherosclerosis, ectopic calcification, and shortened life span (Kuro-o et al., 1997; Kawaguchi et al., 2000; Suga et al., 2000; Mori et al., 2000). Conversely, transgenic mice over-expressing the klotho gene survive longer than wild-type mice (Kurosu et al., 2005). Thus, the klotho gene may function as an aging suppressor gene, extending life span when overexpressed and accelerating aging when inactivated (Kuro-o, 2008). The human homolog of mouse klotho is composed of five exons and extends over $50 \mathrm{~kb}$ on chromosome 13q12. This gene encodes human klotho protein with $86 \%$ identity with the mouse counterpart. Until recently, no premature aging syndromes have been linked to this region (Matsumura et al., 1998). However, recent studies have suggested that a human functional variant of the klotho gene is associated with both reduced human longevity and coronary-artery disease, which may mean that the klotho gene expression also has a role in the development of aging-related phenotypes in humans (Koh et al., 2001; Arking et al., 2002, 2003).

Although klotho protein is predominantly expressed in the kidney, it is detected in a limited number of other tissues, such as the placenta, ovary, prostate gland, and small intestine (Kuro-o et al., 1997; Shiraki-Iida et al., 1998). Intensive studies are being conducted to reveal functions and action mechanisms of this protein. In these studies, the klotho protein is defined as a multi-functional protein involved in several metabolic pathways involving calcium and phosphate homeostasis (Imura et al., 2007; Razzaque, 2009; Alexander et al., 2009), the insulin/insulin-like growth factor 1 (IGF-1) signal pathway (Bartke, 2006), apoptosis (Ikushima et al., 2006), angiotensinII-induced events in the kidney (Negri, 2005), and oxidative stress (Yamamoto et al., 2005). However, it is still unclear what mechanisms are involved in the effects of klotho protein on these pathways.

Regulation of the klotho gene expression is important as much as its function. Induction of the klotho gene could have therapeutic uses in treating aging-related disorders. We examined some trans- and cis-regulatory factors that might have important roles in the regulation of the klotho gene expression.

\section{MATERIAL AND METHODS}

\section{Plasmids}

Plasmids were used to construct protein-expression vectors (p16, p21 and p53) and reporter vectors, pM (Clontech, USA), pTRE-Luc (Clontech), pGFP (Clontech), pSV- $\beta$-Gal 
(Promega, USA), and pCAGGS (Niwa et al., 1991). The plasmids were amplified in Escherichia coli $\mathrm{DH} 5 \alpha$ host bacteria grown in LB ( + amp) liquid media and isolated with a Plasmid Midi Kit (Qiagen, Germany), according to manufacturer instructions.

\section{Cell lines}

A human embryonic kidney (HEK293) cell line was used for transient transfection experiments. The cells were maintained at $37^{\circ} \mathrm{C}, 5 \% \mathrm{CO}_{2}$ and $>90 \%$ relative humidity in Dulbecco's modified Eagle's medium (DMEM), supplemented with $10 \%$ fetal bovine serum (Gibco, Grand Island, NY, USA), $100 \mathrm{U} / \mathrm{mL}$ penicillin and $100 \mu \mathrm{g} / \mathrm{mL}$ streptomycin.

\section{Construction of reporter vectors}

Luciferase- and GFP-reporter vectors were constructed in two-step cloning experiments. First, an intermediate vector containing about $2.1 \mathrm{kbp}$ of the upstream region from the start codon of the human klotho gene was constructed. The klotho gene upstream region was amplified with polymerase chain reaction (PCR) by using 5'-TTGATATCGCGGCCGCTTA AAGCTTTGAGATGCATGT-3' and 5'-ATTACCATGGGCTGCGCGGGAGCCAGGCTC-3' primer pairs, genomic DNA of HEK293 cells as a template, and PrimeSTAR-HS DNA polymerase (TaKaRa, Japan). The PCR product was digested with EcoRV and NcoI (Fermentas, Lithuania), and then NcoI-end was blunted with the Klenow enzyme (Roche, Germany). The pM plasmid was digested with $S m a$ I and $N c o I$, then blunted with Klenow. A large fragment of digested pM was purified from agarose gel and ligated with the PCR amplified upstream region of the human klotho gene with T4-DNA ligase (Roche). The resultant intermediate plasmid was designated as pHKP. The pHKP plasmid was checked with restriction enzyme digestion and then sequenced. The luciferase and GFP ORFs were cloned into the pHKP plasmid. Luciferase coding DNA was derived from pTRE-Luc by digestion with DraI and HindIII, followed by treatment with the Klenow enzyme. GFP coding DNA was prepared from the pGFP plasmid. The pGFP plasmid was first digested with NotI and blunted with Klenow and then digested with NcoI. Luciferase and GFP coding DNA fragments were purified from agarose gel with a DNA extraction kit (Invitrogen, Germany). The pHKP plasmid was digested with $N c o I$ and blunted with the Klenow enzyme and then ligated with luciferase coding DNA fragment. The resultant vector was designed as pHKP-Luc. For the construction of the GFP reporter vector, pHKP-GFP, pHKP was partially digested with $\mathrm{Xba}$ I and blunted with the Klenow enzyme, followed by digestion with $\mathrm{NcoI}$ and then ligated with a GFP coding DNA fragment.

\section{Construction of deleted reporter vectors}

Deleted reporter vectors were derived from pHKP-Luc and pHKP-GFP by digestion with appropriate restriction enzymes. Seven deleted reporter vectors were derived from pHKP-Luc. The pHKP-Luc was digested with NotI/Bgl-II (for pHKP $\Delta \mathrm{I}-\mathrm{Luc}$ ), NotI/ $B a m \mathrm{HI}$ (for pHKP $\Delta \mathrm{II}-\mathrm{Luc}$ ), NotI/SmaI (for pHKP $\Delta \mathrm{III}-\mathrm{Luc}$ ), Bgl-II/BamHI (for $\mathrm{pHKP} \Delta \mathrm{IV}$ Luc), BsmBI (for pHKP $\Delta \mathrm{V}$-Luc), $N d e \mathrm{I} / B g l$-II (for pHKP $\Delta \mathrm{VI}-\mathrm{Luc}$ ), or $N d e \mathrm{I} / B a m \mathrm{HI}$ (for $\mathrm{pHKP} \Delta$ VII-Luc) restriction enzymes and blunted with Klenow. The large DNA fragments 
of digested samples were purified from agarose gel and re-circulated with T4-DNA ligase. Similarly, three deleted GFP reporter vectors were derived from pHKP-GFP by digestion with $N o t \mathrm{I} / B g l$-II (for $\mathrm{pHKP} \Delta \mathrm{I}-\mathrm{GFP}$ ), NotI/BamHI (for $\mathrm{pHKP} \Delta \mathrm{II}-\mathrm{GFP}$ ) and $B g l$-II/BamHI (for pHKP $\triangle \mathrm{IV}$-GFP) restriction enzymes. After treatment with Klenow, the large fragments of digested vectors were purified from agarose gel and re-circulated.

\section{Construction of $\mathrm{p} 16, \mathrm{p} 21$ and $\mathrm{p53}$ expression vectors}

Human p16, p21 and p53 coding DNA fragments were amplified with reverse transcription-PCR. Total RNA was extracted from HEK293 cells with an RNA extraction kit (Roche). cDNA was prepared with reverse transcription by using oligo-dT primers, according to manufacturer instructions. The gene coding regions were amplified form this cDNA with PCR by using 5-TTGAATTCGCCGCCACCATGGAGCCGGCGGCGGGGAGCA-3/5TTGAATTCTCATTTATCATCATCTTTATAATCATCGGGGATGTCTGAGGGACCT-3 (for p16); 5-TTGAATTCGCCGCCACCATGTCAGAACCGGCTGGGGATG-3/5-TTGAATTCT CATTTATCATCATCTTTATAATCGGGCTTCCTCTTGGAGAAGATC-3 (for p21) and 5-TTGAATTCGCCGCCACCATGGAGGAGCCGCAGTCAGATC-3/5-TTGAATTCT CATTTATCATCATCTTTATAATCGTCTGAGTCAGGCCCTTCTGTC-3 (for p53) primer pairs. The PCR products were digested with EcoRI restriction enzyme and purified from agarose gel. pCAGGS plasmid was also digested with EcoRI and dephosphorilated and then ligated with EcoRI-digested PCR products. The resultant vectors were designated as pCAGGS-p16, pCAGGS-p21 and pCAGGS-p53.

\section{Western blot analysis}

p16, p21 and p53 expression was checked with Western blotting. HEK293 cells were seeded onto $6 \mathrm{~cm}$ Petri dishes $\left(2 \times 10^{5}\right.$ cells $)$, incubated under standard culture conditions for $24 \mathrm{~h}$ and transfected with $3 \mu \mathrm{g}$ related expression vectors. After a $48-\mathrm{h}$ incubation, the cells were harvested in $200 \mu \mathrm{L}$ SDS sample buffer. The protein samples were then separated by $15 \%$ SDS-PAGE and transferred onto nitrocellulose filters. The blots were incubated with monoclonal anti-flag antibodies and developed with a chemiluminescent substrate.

\section{Immunofluorescence assay}

HEK293 cells were grown on a glass coverslip in a 24 -well plate and transfected with $0.5 \mu \mathrm{g}$ pCAGGS-p16, pCAGGS-p21 or pCAGGS-p53. At $48 \mathrm{~h}$ post-transfection, cells were fixed with $3 \%$ paraformaldehyde solution, permeabilized with $0.1 \%$ NP-40, washed twice with PBS, and then treated with $1 \%$ skim milk. The cells were incubated with mouse anti-flag at 1:500 dilutions in 1\% skim milk for 60 min and washed twice with 1\% NP-40 and once with PBS. Cells were then stained with FITC-conjugated goat anti-mouse IgG at 1:500 dilutions in $1 \%$ skim milk. The coverslip was washed with $0.1 \%$ NP-40 and mounted in $0.1 \%$ p-phenylenediamine and $80 \%$ glycerol. DNA was stained with propidium iodide. Expression and localization of p16, p21 and p53 in the cells were visualized under a fluorescence microscope. 


\section{DNA transfection}

HEK293 cells were seeded in $24-$ well plates $\left(5 \times 10^{4}\right.$ cell/well) and incubated under standard culture conditions for 20-24 h. The culture medium was renewed with fresh medium $30 \mathrm{~min}$ before transfection. A defined plasmid or a mixture of different plasmids $(0.5-1.2 \mu \mathrm{g}$ for each well) was diluted in DMEM (-) at $20 \mathrm{ng} / \mu \mathrm{L}$ concentrations; $2-3 \mu \mathrm{L}$ FuGENE-HD (Roche) or $4 \mu \mathrm{L}$ DOTAP $(1 \mathrm{mg} / \mathrm{mL}$ in $10 \mathrm{mM}$ HEPES) was added for each $\mu \mathrm{g}$ diluted DNA and incubated for $20 \mathrm{~min}$ at room temperature. The DNA samples were added to cultures and incubated for $48 \mathrm{~h}$. After incubation, the cells were harvested for enzyme assays or inspected under a fluorescent microscope (Olympus BX50) for GFP expression.

\section{Enzyme assays}

Luciferase activity of cell lysates was measured with a luciferase assay kit (Roche), according to manufacturer instructions. Luminescence was detected with a single-tube luminometer (20/20n Single Tube Luminometer-Promega). $\beta$-galactosidase activity of the samples was measured, as previously reported (Turan and Nagata, 2006).

\section{Determination of p16, p21 and p53 effects on human klotho expression}

HEK293 cells were seeded in 24-well plates ( $5 \times 10^{4}$ cell/well) and incubated under standard culture conditions for 20-24 h. The cells grown in each well were transfected with 0.1 $\mu \mathrm{g} \mathrm{pSV}-\beta-\mathrm{Gal}, 0.2 \mu \mathrm{g}$ luciferase reporter vector and increasing amounts of protein expressing vectors. The total amount of plasmid DNAs for each well was equalized with the pCAGGS control vector. At $48 \mathrm{~h}$ post-transfection, the cells were harvested and luciferase and $\beta$-galactosidase activity were measured, as explained above.

\section{Determination of calcium and phosphate effects on human klotho expression}

HEK293 cells were grown in 24-well plates. Five hours before transfection, the medium was changed for DMEM (calcium or phosphate ion free) supplemented with 1\% FBS and different concentrations of $\mathrm{CaCl}_{2}(10,100,300$, or $500 \mu \mathrm{g} / \mathrm{mL})$ or $\mathrm{Na}_{2} \mathrm{HPO}_{4}(10,30,100$, or $300 \mu \mathrm{g} / \mathrm{mL})$. The cells in each well were transfected with $0.2 \mu \mathrm{g} \mathrm{pSV}-\beta$-Gal and $0.5 \mu \mathrm{g}$ luciferase reporter vectors. At $48 \mathrm{~h}$ post-transfection, the cells were harvested and luciferase and $\beta$-galactosidase activities were measured.

\section{Determination of PTH, angiotensin-II and IGF-1 effects on human klotho expression}

Lyophilized parathyroid hormone (PTH, Sigma), IGF-1 (Sigma) and angiotensin-II (Tocris) were re-constituted in sterile distilled water at $50 \mu \mathrm{g} / \mathrm{mL}, 100 \mu \mathrm{g} / \mathrm{mL}$ and $2 \mathrm{mg} / \mathrm{mL}$ final concentrations, respectively, and stored at $-20^{\circ} \mathrm{C}$. HEK293 cells were grown in 24 -well plates under standard growth conditions for $24 \mathrm{~h}$. The medium was changed for DMEM supplied with $1 \%$ FCS and different concentrations of PTH, IGF-1 or angiotensin-II before transfection. The cells were co-transfected with $0.5 \mu \mathrm{g}$ pHKP-Luc reporter vector and $0.2 \mu \mathrm{g} \mathrm{pSV- \beta -Gal} \mathrm{as} \mathrm{a}$ control. At $48 \mathrm{~h}$ post-transfection, the cells were harvested and luciferase and $\beta$-galactosidase activities were measured. 


\section{RESULTS}

\section{Reporter gene expression under the control of human klotho upstream region}

To identify the possible cis- and trans-acting regulatory elements affecting the klotho gene transcription, upstream deleted reporter vectors were derived from $\mathrm{pHKP}$ Luc and pHKP-GFP by restriction enzyme digestion (Figure 1). These constructs were transfected into HEK293 cells grown in 24-well plates. Each reporter vector was cotransfected with pSV- $\beta$-Gal plasmid as a control. At $48 \mathrm{~h}$ post-transfection, luciferase and $\beta$-galactosidase activities in cell lysates were quantified. Luciferase activity was normalized with $\beta$-galactosidase. The samples showing the lowest relative luciferase activity (that is transfected with pHKP-Luc) were designated as 1 , and the activities of other samples were calculated as a function of that activity (Figure 2). Deletion of some parts of the klotho gene upstream significantly induce reporter gene expression. Samples transfected with pHKP $\triangle$ II-Luc had the highest luciferase activity, which was 50-60-fold higher than with pHKP-Luc. The second-highest activity was detected in cells transfected with pHKP $\triangle$ III-Luc, the other constructs yielded a 2- to 8-fold increase in luciferase activity compared to pHKP-Luc.

Quantitave results obtained with luciferase expressing vectors were confirmed with GFP reporter (Figure 3). The highest rate of GFP expression was observed in HEK293 cells transfected with pHK $\Delta$ II-GFP.

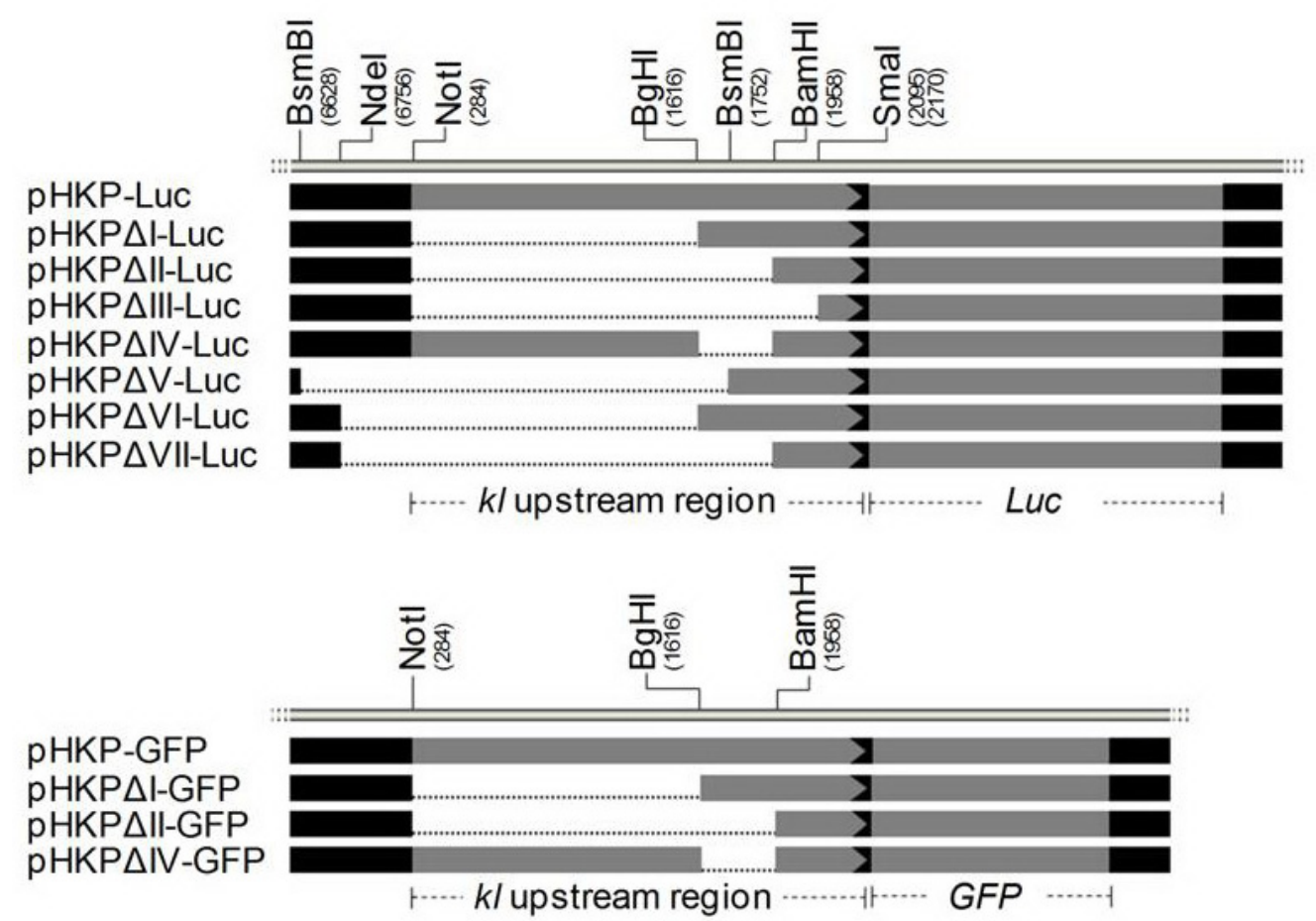

Figure 1. A serial of reporter vectors with deletions in the human klotho $(k l)$ upstream region. 


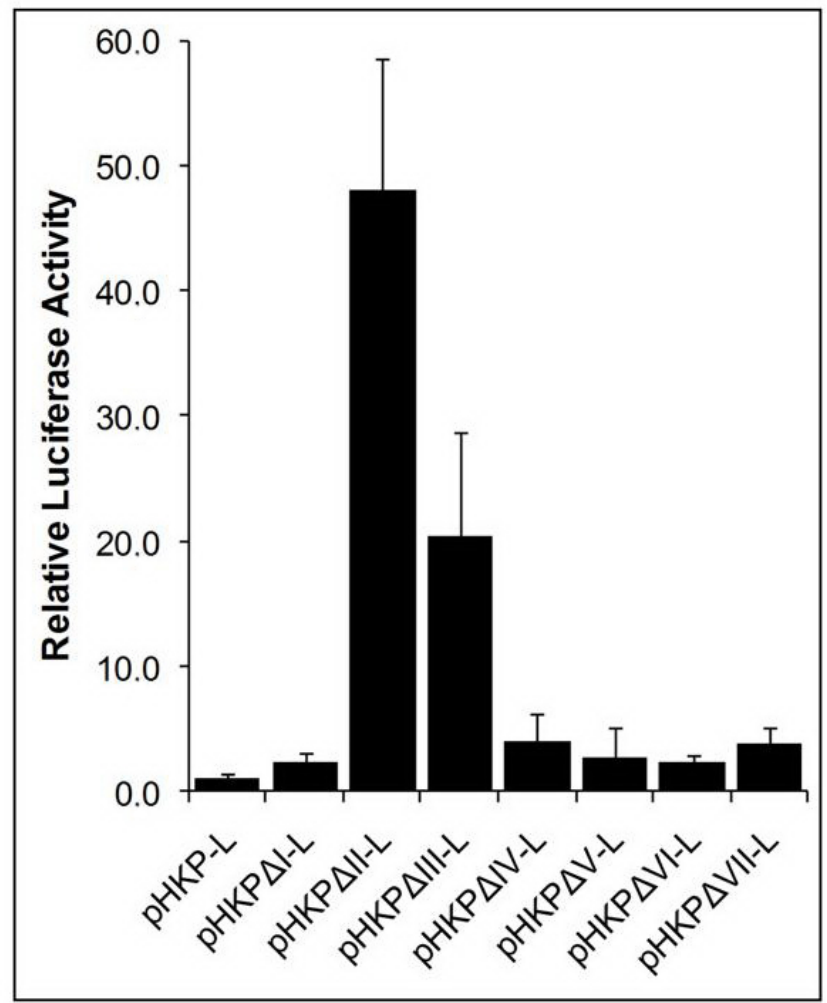

Figure 2. The relative luciferase activities in HEK293 cells transfected with full-length or serial deletion constructs. The cells were grown in 24-well plates and co-transfected with $0.5 \mu \mathrm{g}$ luciferase reporter vector and $0.2 \mu \mathrm{g} \mathrm{pSV}-\beta$ Gal. Error bars represent standard deviations $(\mathrm{N}=5)$.
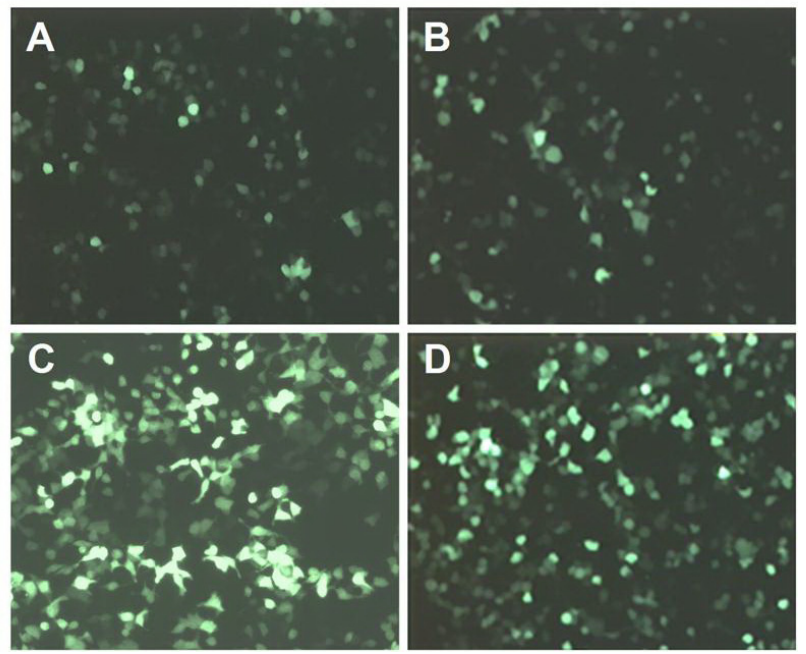

Figure 3. GFP expression in HEK293 cells transfected with $0.5 \mu \mathrm{g}$ of A. pHKP-GFP; B. pHKP $\Delta \mathrm{I}-\mathrm{GFP}$; C. pHKP $\triangle \mathrm{II}-\mathrm{GFP}$, or D. pHKP $\Delta \mathrm{IV}-\mathrm{GFP}$. 


\section{Effect of p16, p21 and p53 on luciferase reporter gene expression}

To determine the effect of apoptosis-related proteins on human klotho expression in HEK293 cells, we constructed vector plasmids pCAGGS-p16, pCAGGS-p21 and pCAGGSp53, expressing p16, p21 and p53, respectively. Expression and localization of these proteins in HEK293 cells were demonstrated with Western blotting and immunofluorescence assays (Figures 4 and 5). p16 protein tagged with flag had a cytoplasmic localization. In contrast, p21 and p53 proteins were located in the nucleus. The effects of p16, p21 and p53 proteins on the klotho promoter-driven luciferase gene expression were investigated in transiently transfected cells. The cells, grown in a 24-well plate, were co-transfected with $0.2 \mu \mathrm{g}$ pHKP-Luc reporter vector, $0.1 \mu \mathrm{g} \mathrm{pSV}-\beta-\mathrm{Gal}$ and $0.01-0.3 \mu \mathrm{g}$ protein-expressing vectors. At $48 \mathrm{~h}$ posttransfection, the cells were harvested and analyzed for luciferase and $\beta$-galactosidase activity. Luciferase activities were normalized with $\beta$-galactosidase and compared to those of nontransfected cells (Figure 6). The p16 and p53 proteins inhibited luciferase expression under control of the klotho promoter in a dose-dependent manner. In contrast, luciferase activity was not significantly affected by different expression levels of $\mathrm{p} 21$.

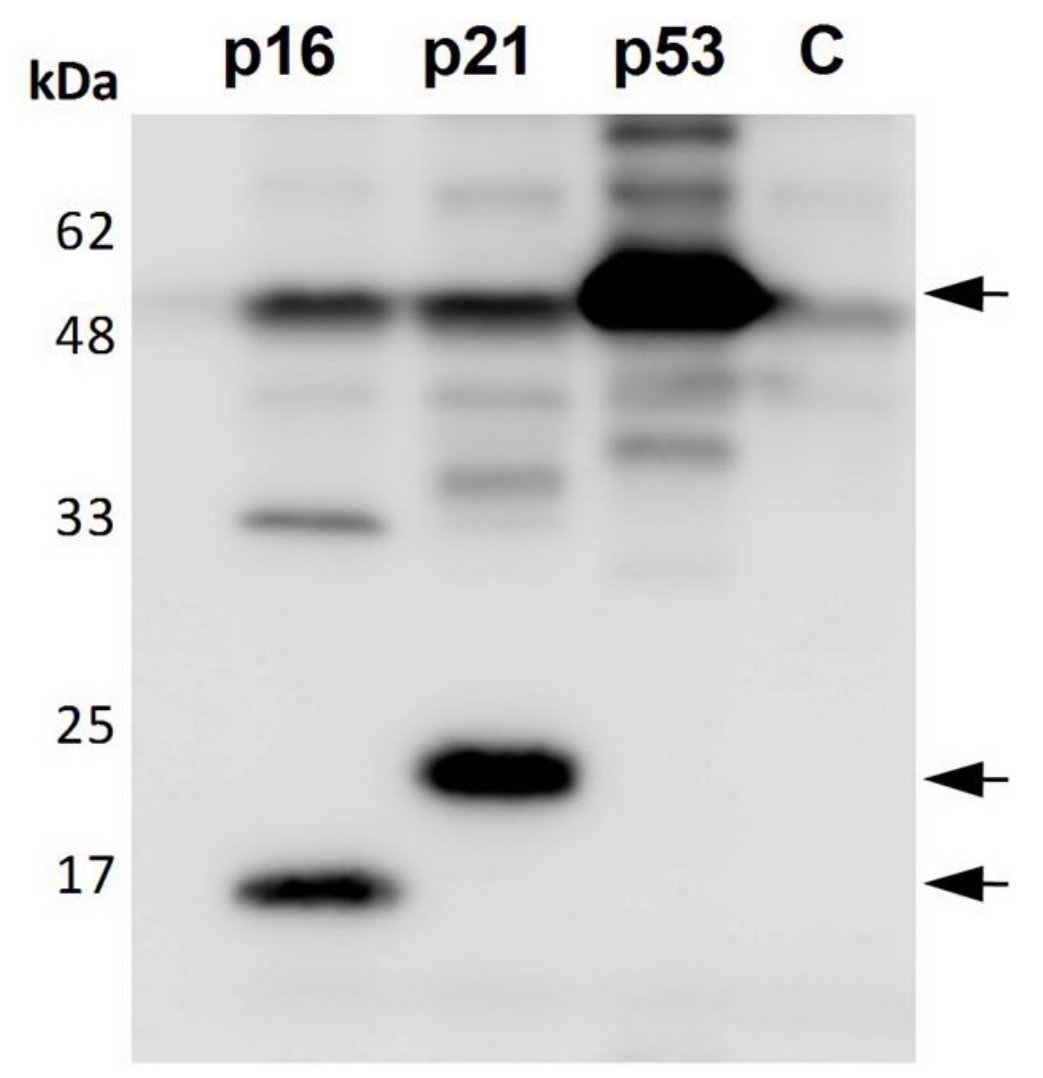

Figure 4. Western blot analysis of p16, p21 and p53 expression in HEK233 cells. The cells were grown on 6-cm Petri dishes and transiently transfected with $3 \mu \mathrm{g}$ pCAGGS-p16, pCAGGS-p21 or pCAGGS-p53. The proteins tagged with the flag epitope were visualized with monoclonal anti-flag antibodies. $\mathrm{C}=$ non-transfected cell lysate. 

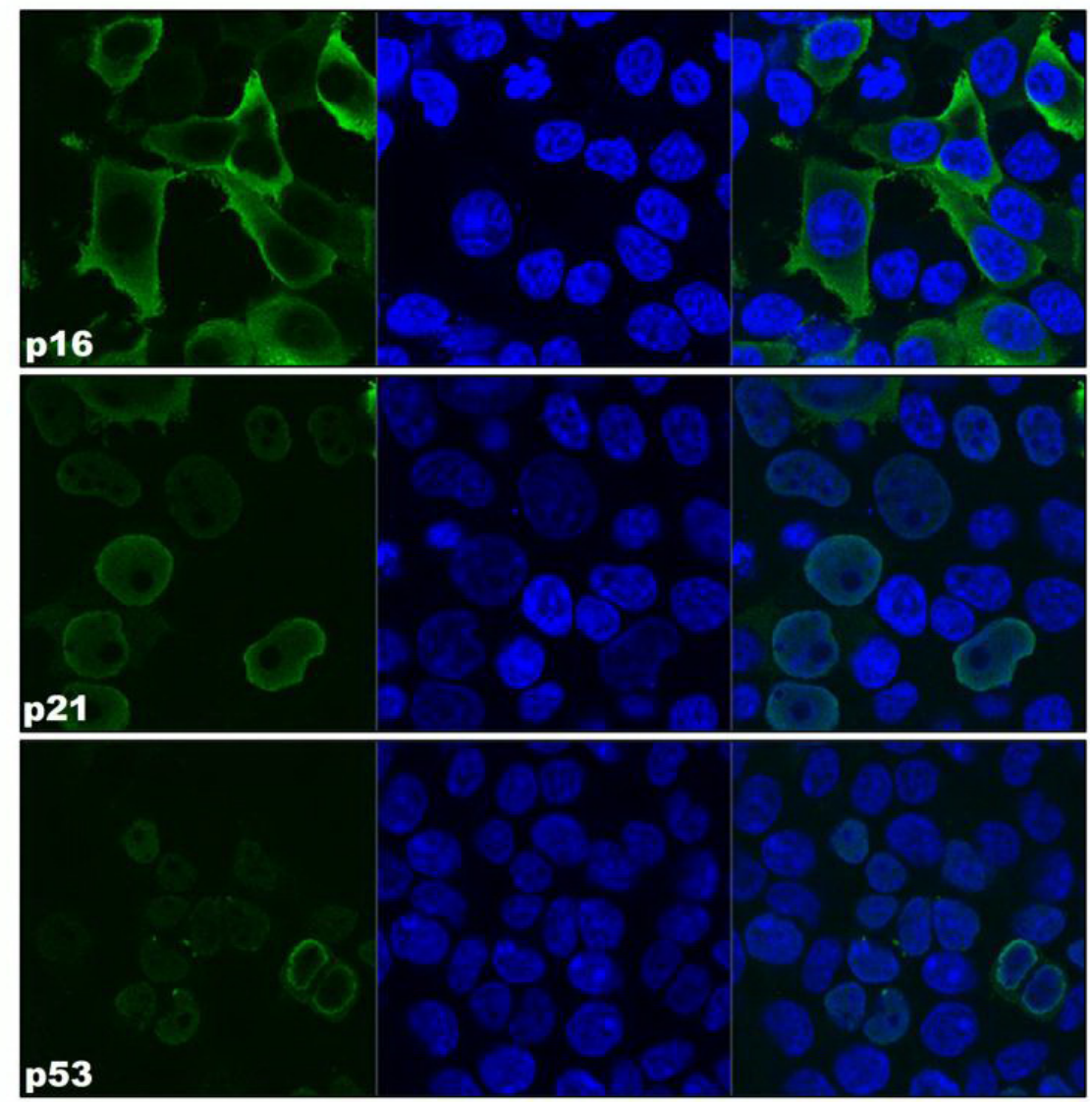

Figure 5. Expression and localization of p16, p21 and p53 proteins in HEK293 cells.

\section{Effect of calcium and phosphate ions on luciferase gene expression}

The klotho protein affects $\mathrm{Ca}$ and P/vitamin D homeostasis (Nabeshima, 2008). In contrast, the effect of these ions on the klotho gene expression is unclear. Here, we investigated the effect of $\mathrm{Ca}$ and $\mathrm{P}$ ions on human klotho expression by using luciferase reporter vectors. The experiments were carried out with HEK293 cells grown in DMEM supplied with 1\% FCS and different concentrations of $\mathrm{Ca}$ and $\mathrm{P}$ ions. The cells were co-transfected with $0.5 \mu \mathrm{g}$ reporter vector and $0.2 \mu \mathrm{g} \mathrm{pSV- \beta -Gal.} \mathrm{After} \mathrm{a} \mathrm{48-h} \mathrm{incubation} \mathrm{under} \mathrm{standard} \mathrm{culture} \mathrm{condi-}$ tions, the cells were harvested and the relative luciferase activities of cell lysates were calculated (Figure 7). Both ions were tested on pHKP-Luc and pHKP $\Delta I I-L u c$ vectors. In contrast to p16 and p53 proteins, $\mathrm{Ca}$ and $\mathrm{P}$ ions had strong stimulatory effects on luciferase expression, compared to $\beta$-galactosidase, which is driven by the SV40 promoter. On the other hand, over a certain level of ion concentrations, there was a tendency of a decrease in the klotho gene expression. Higher luciferase activity was obtained with $\mathrm{pHKP} \Delta \mathrm{II}-\mathrm{Luc}$ reporter compared to the pHKP-Luc vector in the presence of $\mathrm{Ca}$ ions. When $\mathrm{P}$ ions were tested at the same concentration, the pHKP-Luc reporter vector gave the highest level of luciferase activity. 

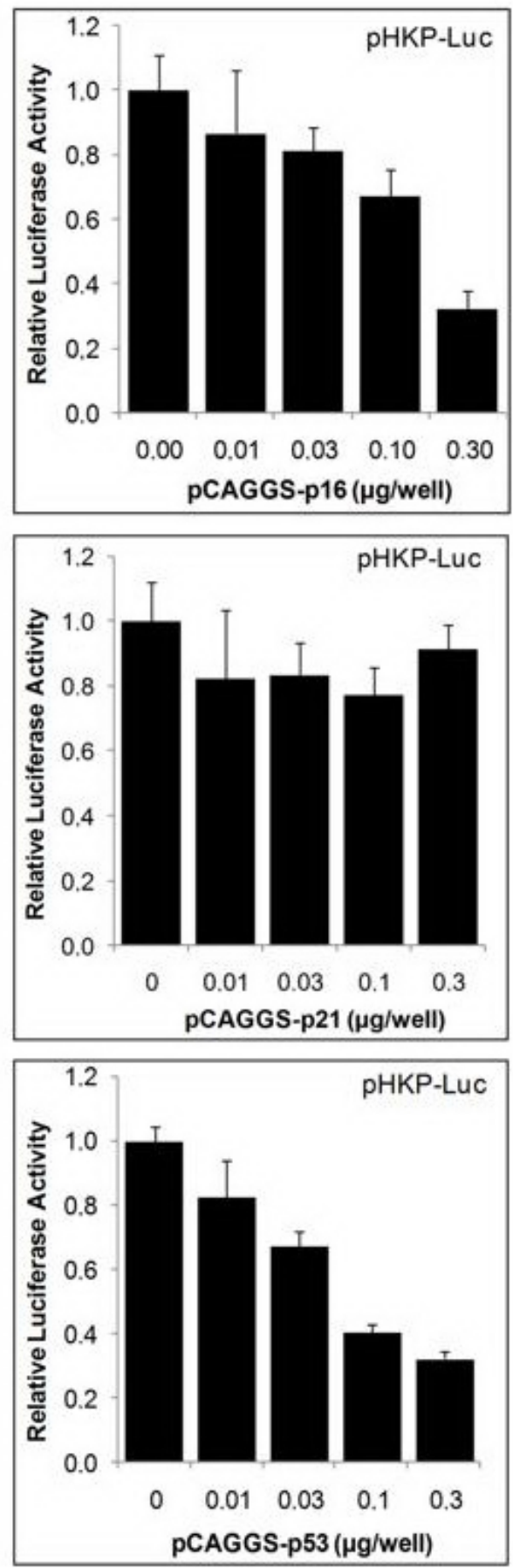

Figure 6. Relative luciferase activities in HEK293 expressing increasing amounts of p16, p21 or p53 protein. Error bars represent standard deviations $(\mathrm{N}=3)$. 

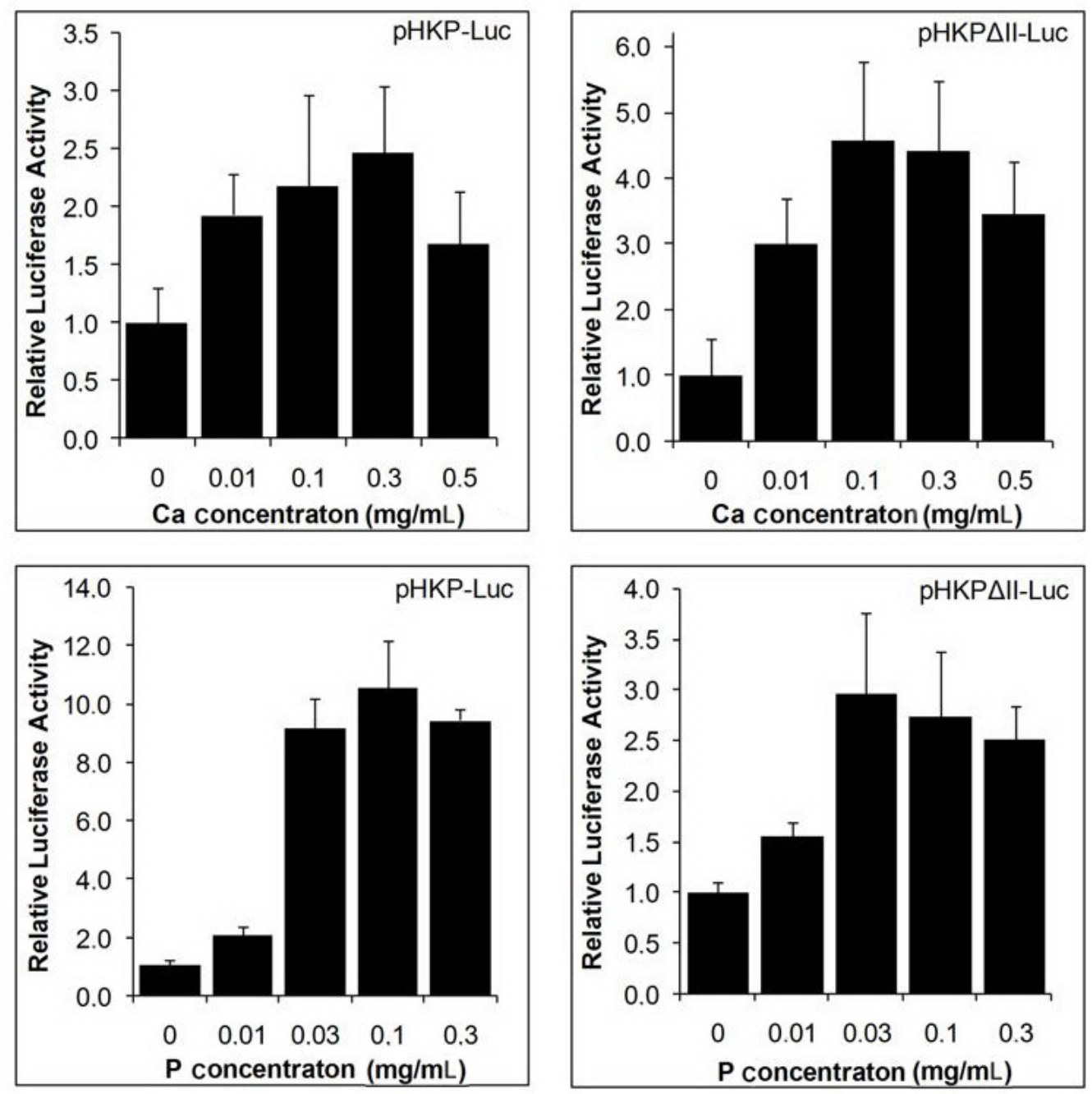

Figure 7. The effects of calcium and phosphate ions on luciferase reporter gene expression in HEK293 cells. The cells were grown in 24-well plates containing complete medium for $20 \mathrm{~h} ; 4 \mathrm{~h}$ before transfection the medium was changed to DMEM (calcium- or phosphate-free) supplied with 1\% FCS and defined concentrations of $\mathrm{CaCl}_{2}$ or $\mathrm{NaH}_{2} \mathrm{PO}_{4}$. Error bars represent standard deviations $(\mathrm{N}=3)$.

\section{Effect of PTH, IGF-1 and angiotensin-II on luciferase gene expression}

The klotho protein is involved in endocrine regulation of mineral metabolism and some signaling factors, such as insulin, IGF-1, angiotensin-II, and PTH. However, possible relationships such as negative/positive feedback mechanisms between the klotho protein and some signaling factors or hormones remain to be determined. Here, we examined the effect of some extracellular protein factors on the klotho expression. We tested PTH, IGF-1 and angiotensin-II effects on the klotho expression in HEK293 cells. The cells were grown in DMEM 
supplied with $1 \%$ FCS and with increasing concentrations of hormone or growth factors and transfected with pHKP-Luc vector. At 48 h post-transfection, luciferase gene expression was defined in cell lysates (Figure 8). Luciferase activity was not significantly changed in the presence of PTH, IGF-1 or angiotensin-II at different concentrations.
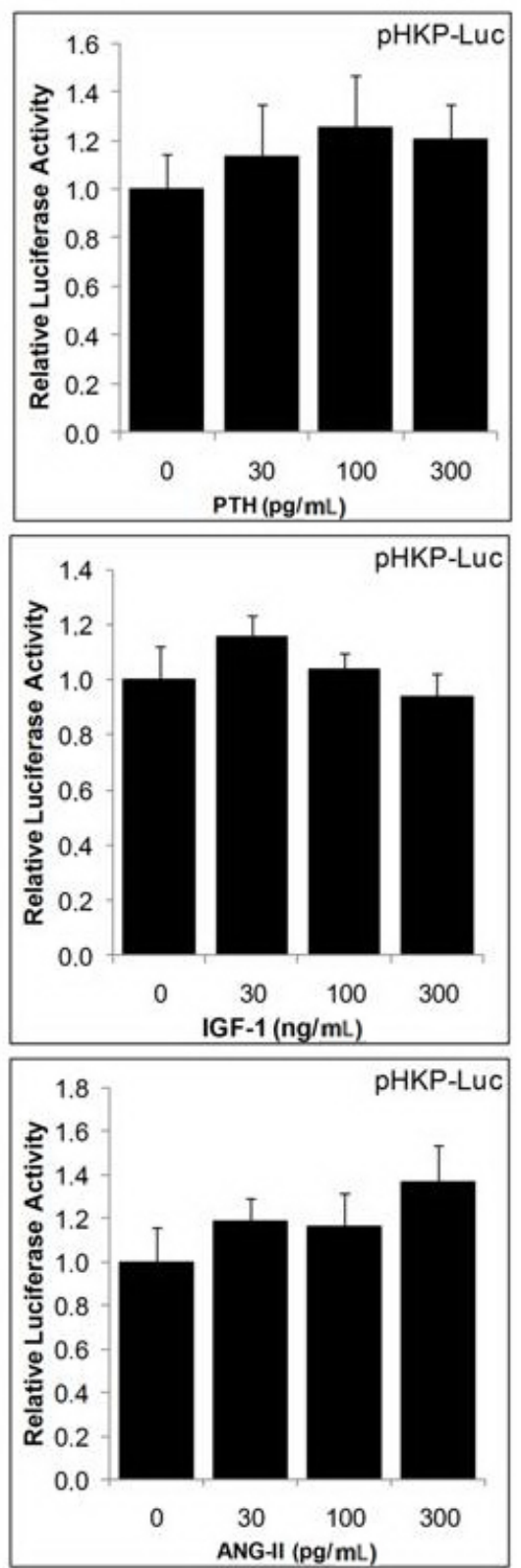

Figure 8. The effects of PTH, IGF-1 and angiotensin-II on human klotho expression in HEK292 cells. Error bars represent standard deviations $(\mathrm{N}=3)$. 


\section{DISCUSSION}

The klotho protein is multifunctional and is involved in several signal pathways and in ion homeostasis (Imura et al., 2007; Razzaque, 2009; Alexander et al., 2009). However, the mechanism by which klotho affects these processes has remained unclear. The stimulatory or inhibitory effects of cis- and trans-regulatory factors on the klotho gene expression are also not completely understood. In this context, we investigated the effects of some intra- and extracellular factors on human klotho expression. We constructed pHKP-Luc reporter vector containing about $2.1 \mathrm{kbp}$ upstream of human klotho and derived several reporter vectors from pHKP-Luc with restriction enzyme digestions (Figure 1). These constructs were transfected into HEK293 cells and the reporter gene (luciferase) activity was measured (Figure 2). An approximately 50 -fold increase in reporter activity was obtained with pHKP $\Delta \mathrm{II}-\mathrm{Luc}$ compared to that with pHKP-Luc. pHKP $\triangle \mathrm{I}-\mathrm{Luc}$, which has an extra 342-bp fragment located between $B g l \mathrm{II}$ and $B a m \mathrm{HI}$, resulted in a 2.5-fold increase in luciferase activity. This result suggested that regulatory motifs located between $B g l \mathrm{II}$ and $B a m \mathrm{HI}$ restriction sites down-regulate the klotho gene expression. In contrast, pHKP $\Delta$ IV-Luc with a 342-bp deletion between BglII and BamHI, gave about 4-fold higher expression than pHKP-Luc, but unexpectedly lower expression than pHKP $\Delta$ II-Luc. An extra 200-bp deletion between NdeI and NotI of pHKPAIILuc (resultant vector pHKP $\Delta$ VII-Luc) also greatly reduced reporter activity. The analysis of 342-bp BglII/BamHI for transcription factor binding sites with a software (TRES Transcription Regulatory Element Search) indicated mammalian PD1 silencer resembling motifs (..CCAAAATTAT..........TATTAAAA..; Weissman and Singer, 1991) in this region. An approximately 4-fold increase in reporter activity was observed in HEK293 cells transfected with reporter vectors lacking from this region. This result suggests that PD1 silencer resembling motifs down-regulate the klotho expression. However, high luciferase activity of pHKP $\Delta$ II-Luc is not explained only by silencer motifs. Enhancer motifs located between NdeI and NotI restriction sites that belong to the vector backbone have a stimulatory effect on reporter gene expression.

p16 is a key tumor suppressor that blocks the progression of the cell cycle by binding to either CDK4 or CDK6 (Serrano et al., 1993). This protein is also known as p16 $6^{\mathrm{INK} 4 \mathrm{a}}$, INK4 or CDK4I. Expression of p16 has dramatically increase with aging in most rodents, and in human tissues (Melk et al., 2003, 2004; Liu et al., 2009). Here we showed that overexpression of the p16 protein inhibits reporter luciferase expression in a dose-dependent manner. This suggests that the p16 has a down-regulatory effect on human klotho expression. However, more data will be needed to confirm that p16 has a role in aging by affecting the klotho gene expression. Another important tumor suppressor protein is p53. Key functions of p53 are induction of cell cycle arrest, senescence, and apoptosis in response to DNA damage (Abdulamir et al., 2008). $\mathrm{p} 21$, another apoptosis-related protein, is up-regulated by p53 in response to DNA damage in the apoptotic pathway (Abdulamir et al., 2008). It was shown that klotho knock down MRC-5 cells had decreased p53 synthesis and stimulated replication and cell division. This suggests that the klotho protein affects apoptosis via the p53/p21 pathway (de Oliveira, 2006). However, the role of p53/p21 in the klotho expression is unclear. To look for clues concerning the klotho gene regulation, we tested the effect of these proteins on the klotho expression. p53 has inhibitory effects on luciferase reporter expression in a dose-dependent manner, as does p16 (Figure 6). In contrast to $\mathrm{p} 16$ and $\mathrm{p} 53$ proteins, $\mathrm{p} 21$ did not have significant effects on the klotho expression. This finding suggests that p53 might down-regulate the klotho gene independent of the p53/p21 pathway. 
The klotho gene codes a single-pass transmembrane protein with a large extracellular domain. The extracellular domain of this protein is clipped on the cell surface by membraneanchored proteases to generate a secreted form. Thus, the klotho protein exists in at least two forms. One is membrane-bound klotho, and the other is secreted klotho, which exists in the blood, urine, and cerebral spinal fluid (Matsumura et al., 1998; Kuro-o, 2010). These two forms of klotho have distinct functions. Membrane-bound klotho interacts with fibroblast growth factor (FGF) receptors to form high-affinity receptors for FGF23. Secreted klotho protein functions as a humoral factor that regulates several ion channels and transporters, and other processes, including insulin and IGF-1 signaling (Kuro-o, 2006). In cooperation with Fgf23, it regulates calcium concentration by suppression of $1,25(\mathrm{OH}) 2 \mathrm{D}$ synthesis, and reabsorption of phosphate in the distal convoluted part of the nephron (Ikushima et al., 2006). These reports suggest that the klotho gene expression may be regulated by $\mathrm{Ca}$ and $\mathrm{P}$ ions. Here, we detected that both $\mathrm{Ca}$ and $\mathrm{P}$ ions stimulate reporter luciferase activity under the control of human klotho promoter in HEK293 cells in a dose-dependent manner (Figure 7). On the other hand, at concentrations of $0.3 \mathrm{mg} / \mathrm{mL}$ or more, $\mathrm{Ca}$ and $\mathrm{P}$ ions negatively affect the klotho expression. In a previous report, it was shown that klotho mRNA in bovine parathyroid cells was inversely correlated with serum calcium levels (Bjorklund et al., 2008). At first glance, this result seems to contradict our conclusions. In that report, an inhibitory effect of $\mathrm{Ca}$ ions at values higher than $1.5 \mathrm{mM}$ was detected. However, in our study, the klotho gene expression was stimulated at Ca concentrations that were lower than $1.5 \mathrm{mM}$. These results lead us to assume that $\mathrm{Ca}$ (and also P) ions stimulate the klotho gene expression until a certain threshold value, but they have an inhibitory effect over this threshold.

We also tested the effects of the extracellular factors PTH, IGF-1 and angiotensin-II on the human klotho gene expression because of their relationship with the klotho protein. Recent reports showed that the klotho protein can act as a hormone and inhibits the intracellular insulin/IGF-1 signaling cascade (Wolf et al., 2008). The klotho protein also inhibits PTH secretion indirectly through the action of FGF23. We showed that none of these factors have a significant up- or down-regulatory function on the klotho expression in HEK293 cells (Figure 8). However, one has to consider the serum content of growth medium as well as the possible insensitivity of HEK293 cells to the factors that were tested.

We conclude that the human klotho gene expression is under strict control of cis- and trans-acting factors. Overexpression of p16 and p53 tumor suppressor proteins in HEK293 cells decreases the klotho expression. In contrast, increasing concentrations of calcium and phosphate ions stimulate the klotho expression.

\section{ACKNOWLEDGMENTS}

Research supported by a grant from the Scientific and Technical Research Council of Turkey (Grant \#SBAG-108S293) and by the Marmara University Research Foundation (Grant \#SAG-A-040609-0168).

\section{REFERENCES}

Abdulamir AS, Hafidh RR, Mahdi LK, Al-jeboori TR, et al. (2008). The interplay between p53 and p21 tumor suppressor proteins in the transformation of colorectal adenoma to carcinoma. Am. J. Immunol. 4: 14-22.

Alexander RT, Woudenberg-Vrenken TE, Buurman J, Dijkman H, et al. (2009). Klotho prevents renal calcium loss. J. Am. Soc. Nephrol. 20: 2371-2379.

Arking DE, Krebsova A, Macek M Sr, Macek M Jr, et al. (2002). Association of human aging with a functional variant of 
klotho. Proc. Natl. Acad. Sci. U. S. A. 99: 856-861.

Arking DE, Becker DM, Yanek LR, Fallin D, et al. (2003). Klotho allele status and the risk of early-onset occult coronary artery disease. Am. J. Hum. Genet. 72: 1154-1161.

Bartke A (2006). Long-lived Klotho mice: new insights into the roles of IGF-1 and insulin in aging. Trends Endocrinol. Metab. 17: 33-35.

Bjorklund P, Krajisnik T, Akerstrom G, Westin G, et al. (2008). Type I membrane klotho expression is decreased and inversely correlated to serum calcium in primary hyperparathyroidism. J. Clin. Endocrinol. Metab. 93: 4152-4157.

de Oliveira RM (2006). Klotho RNAi induces premature senescence of human cells via a p53/p21 dependent pathway. FEBS Lett. 580: 5753-5758.

Ikushima M, Rakugi H, Ishikawa K, Maekawa Y, et al. (2006). Anti-apoptotic and anti-senescence effects of Klotho on vascular endothelial cells. Biochem. Biophys. Res. Commun. 339: 827-832.

Imura A, Tsuji Y, Murata M, Maeda R, et al. (2007). Alpha-klotho as a regulator of calcium homeostasis. Science 316: 1615-1618.

Kawaguchi H, Manabe N, Chikuda H, Nakamura K, et al. (2000). Cellular and molecular mechanism of low-turnover osteopenia in the klotho-deficient mouse. Cell. Mol. Life Sci. 57: 731-737.

Koh N, Fujimori T, Nishiguchi S, Tamori A, et al. (2001). Severely reduced production of klotho in human chronic renal failure kidney. Biochem. Biophys. Res. Commun. 280: 1015-1020.

Kuro-o M (2006). Klotho as a regulator of fibroblast growth factor signaling and phosphate/calcium metabolism. Curr. Opin. Nephrol. Hypertens. 15: 437-441.

Kuro-o M (2008). Klotho as a regulator of oxidative stress and senescence. Biol. Chem. 389: 233-241.

Kuro-o M (2010). Klotho. Pflugers Arch. 459: 333-343.

Kuro-o M, Matsumura Y, Aizawa H, Kawaguchi H, et al. (1997). Mutation of the mouse klotho gene leads to a syndrome resembling ageing. Nature 390: 45-51.

Kurosu H, Yamamoto M, Clark JD, Pastor JV, et al. (2005). Suppression of aging in mice by the hormone Klotho. Science 309: 1829-1833.

Liu Y, Sanoff HK, Cho H, Burd CE, et al. (2009). Expression of p16 $6^{(\mathrm{INK} 4 a)}$ in peripheral blood T-cells is a biomarker of human aging. Aging Cell 8: 439-448.

Matsumura Y, Aizawa H, Shiraki-Iida T, Nagai R, et al. (1998). Identification of the human klotho gene and its two transcripts encoding membrane and secreted klotho protein. Biochem. Biophys. Res. Commun. 242: 626-630.

Melk A, Kittikowit W, Sandhu I, Halloran KM, et al. (2003). Cell senescence in rat kidneys in vivo increases with growth and age despite lack of telomere shortening. Kidney Int. 63: 2134-2143.

Melk A, Schmidt BM, Takeuchi O, Sawitzki B, et al. (2004). Expression of p16 ${ }^{\mathrm{INK} 4 a}$ and other cell cycle regulator and senescence associated genes in aging human kidney. Kidney Int. 65: 510-520.

Mori K, Yahata K, Mukoyama M, Suganami T, et al. (2000). Disruption of klotho gene causes an abnormal energy homeostasis in mice. Biochem. Biophys. Res. Commun. 278: 665-670.

Nabeshima Y (2008). The discovery of alpha-Klotho and FGF23 unveiled new insight into calcium and phosphate homeostasis. Cell. Mol. Life Sci. 65: 3218-3230.

Negri AL (2005). The klotho gene: a gene predominantly expressed in the kidney is a fundamental regulator of aging and calcium/phosphorus metabolism. J. Nephrol. 18: 654-658.

Niwa H, Yamamura K and Miyazaki J (1991). Efficient selection for high-expression transfectants with a novel eukaryotic vector. Gene 108: 193-199.

Razzaque MS (2009). The FGF23-Klotho axis: endocrine regulation of phosphate homeostasis. Nat. Rev. Endocrinol. 5: 611-619.

Serrano M, Hannon GJ and Beach D (1993). A new regulatory motif in cell-cycle control causing specific inhibition of cyclin D/CDK4. Nature 366: 704-707.

Shiraki-Iida T, Aizawa H, Matsumura Y, Sekine S, et al. (1998). Structure of the mouse klotho gene and its two transcripts encoding membrane and secreted protein. FEBS Lett. 424: 6-10.

Suga T, Kurabayashi M, Sando Y, Ohyama Y, et al. (2000). Disruption of the klotho gene causes pulmonary emphysema in mice. Defect in maintenance of pulmonary integrity during postnatal life. Am. J. Respir. Cell Mol. Biol. 22: 26-33.

Turan K and Nagata K (2006). Chitosan-DNA nanoparticles: the effect of cell type and hydrolysis of chitosan on in vitro DNA transfection. Pharm. Dev. Technol. 11: 503-512.

Weissman JD and Singer DS (1991). Striking similarities between the regulatory mechanisms governing yeast matingtype genes and mammalian major histocompatibility complex genes. Mol. Cell. Biol. 11: 4228-4234.

Wolf I, Levanon-Cohen S, Bose S, Ligumsky H, et al. (2008). Klotho: a tumor suppressor and a modulator of the IGF-1 and FGF pathways in human breast cancer. Oncogene 27: 7094-7105.

Yamamoto M, Clark JD, Pastor JV, Gurnani P, et al. (2005). Regulation of oxidative stress by the anti-aging hormone klotho. J. Biol. Chem. 280: 38029-38034. 